\title{
Two-loop electroweak Sudakov logarithms for massive fermion scattering
}

\author{
Ansgar Denner, Bernd Jantzen \\ Paul Scherrer Institut, CH-5232 Villigen PSI, Switzerland \\ E-mail: Ansgar.Denner@psi.ch, physics@bernd-jantzen.de \\ Stefano Pozzorini* \\ Max-Planck-Institut für Physik, Föhringer Ring 6, D-80805 München, Germany \\ E-mail: pozzorinemppmu.mpg.de
}

\begin{abstract}
We study the asymptotic behaviour of two-loop electroweak corrections at energies $Q^{2} \gg M_{\mathrm{W}}^{2}$, where logarithms of the type $\ln \left(Q^{2} / M_{\mathrm{W}}^{2}\right)$ become dominant. The calculation of the leading and next-to-leading logarithmic terms for massless and massive fermion-scattering processes is summarized. The derivations are performed diagrammatically within the spontaneously broken electroweak theory. We find that the soft and collinear singularities resulting from photons can be factorized into a QED-like term and that, up to logarithms of the Z-W mass ratio, the effects of symmetry breaking cancel. This result supports resummation prescriptions that are based on a symmetric $\mathrm{SU}(2) \times \mathrm{U}(1)$ theory matched with QED at the electroweak scale.
\end{abstract}

8th International Symposium on Radiative Corrections

October 1-5, 2007

Florence, Italy

\footnotetext{
* Speaker.
} 


\section{Introduction}

At $\mathrm{TeV}$ colliders, the electroweak corrections are strongly enhanced by logarithms of the form $\alpha^{l} \ln ^{j}\left(Q^{2} / M^{2}\right)$, with $M=M_{\mathrm{W}} \sim M_{\mathrm{Z}}$ and $j \leq 2 l$. These logarithmic corrections affect every reaction that involves electroweakly interacting particles and is characterized by scattering energies $Q^{2} \gg M^{2}$. For $Q \sim 1 \mathrm{TeV}$ their impact can reach tens of per cent at one loop and several per cent at two loops (see for instance Refs. [1,2] and references therein). Their origin is twofold. The renormalization of UV singularities at the scale $\mu_{\mathrm{R}} \lesssim M$ gives rise to terms of the form $\ln \left(Q^{2} / \mu_{\mathrm{R}}^{2}\right)$. In addition, the interactions of the initial- and final-state particles with soft and/or collinear gauge bosons give rise to mass-singular logarithms, i.e. $\ln \left(Q^{2} / M^{2}\right)$ terms that are formally singular in the limit $M \rightarrow 0$. Since they originate from UV, soft and collinear singularities, electroweak logarithmic corrections have universal properties that can be studied in a process-independent way and exhibit interesting analogies with QCD. At one loop, the leading logarithms (LLs) and next-toleading logarithms (NLLs) factorize and are described by a general formula that applies to arbitrary Standard-Model processes [3,4]. The properties of electroweak logarithmic corrections beyond one loop are investigated with two complementary approaches in the literature: (i) Evolution equations, which are well known in QED and QCD, are applied to the electroweak theory in order to obtain the higher-order terms through a resummation [5-9]. In this approach the evolution is split into two regimes, where the electroweak interaction is described in terms of $S U(2) \times U(1)$ and $U_{e m}(1)$ symmetric gauge theories. ${ }^{1}$ This splitting is assumed to correctly reproduce all relevant implications of electroweak symmetry breaking in LL, NLL and NNLL approximation. (ii) This assumption and the resulting resummation prescriptions can be checked and improved by means of explicit diagrammatic two-loop calculations based on the (spontaneously broken) electroweak Lagrangian. So far, all existing diagrammatic results are in agreement with the resummation prescriptions, however up to now only a small subset of logarithms and processes has been computed explicitly. While the LLs [11] and the angular-dependent subset of the NLLs [12] have been derived for arbitrary processes, complete diagrammatic calculations at (or beyond) the NLL level exist only for matrix elements involving massless external fermions [13-15]. In the literature, no explicit NLL calculation exists for reactions involving massive scattering particles.

In the following we present a recent two-loop NLL calculation [16] for $n$-fermion processes $f_{1} f_{2} \rightarrow f_{3} \ldots f_{n}$ involving an arbitrary number of leptons and/or quarks. While all fermions with $m_{f} \ll M$ are treated as massless, the top-quark mass effects are taken into account. For all invariants $r_{i j}=\left(p_{i}+p_{j}\right)^{2}$ we assume $\left|r_{i j}\right| \sim Q^{2} \gg M^{2}$, and the differences between the W, Z, H and t mass are taken into account. Soft and collinear singularities from massless virtual photons are regularized dimensionally and arise as $\varepsilon$-poles in $D=4-2 \varepsilon$ dimensions. For consistency, the same power counting is applied to $\ln \left(Q^{2} / M^{2}\right)$ and $1 / \varepsilon$ singularities. Thus, in NLL approximation we include all $\varepsilon^{-k} \ln ^{j-k}\left(Q^{2} / M^{2}\right)$ terms with total power $j=2,1$ at one loop and $j=4,3$ at two loops. The photonic singularities are factorized in a gauge-invariant electromagnetic term. The remaining part of the corrections - which is finite, gauge invariant, and does not depend on the scheme adopted to regularize photonic singularities - contains only $\ln \left(Q^{2} / M^{2}\right)$ terms. The divergences contained in the electromagnetic term cancel if real-photon emission is included.

\footnotetext{
${ }^{1}$ A new approach based on soft-collinear effective theory has been developed in Ref. [10].
} 


\section{Extraction of soft, collinear and UV contributions from Feynman diagrams}

Let us consider the two-loop diagrams that produce NLLs within the 't Hooft-Feynman gauge. These diagrams are obtained from the LL one-loop diagrams, i.e. diagrams with external-leg exchange of soft-collinear gauge bosons, via insertions of one-loop sub-diagrams that produce an additional logarithm. We start with the diagrams that do not contain Yukawa interactions,
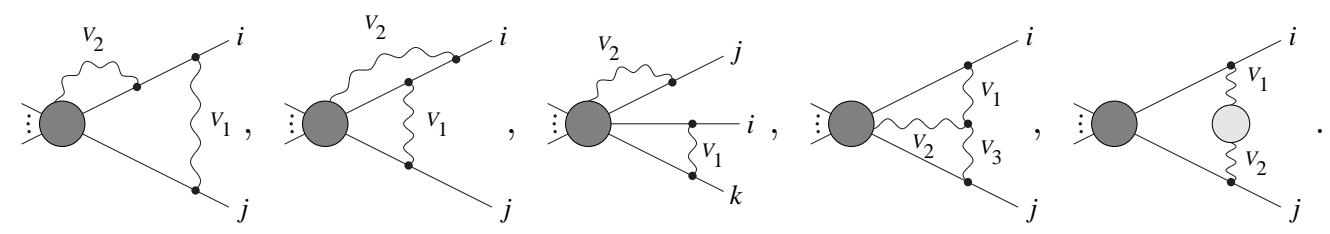

Here $V_{i}=A, Z, W^{ \pm}$, and the external lines $i, j, k=1, \ldots, n$ are massless and/or massive fermions. In the first four diagrams in (2.1) the gauge boson $V_{2}$ can couple to an external line or to an internal propagator. In the latter case the only relevant region is the one where $V_{2}$ is collinear to an external fermion and, using collinear Ward identities, we find that this type of contributions cancel $[15,16]$. The remaining diagrams contain a tree sub-diagram that corresponds to the original process and depends only on the external momenta. ${ }^{2}$ These diagrams are called factorizable, since their NLL contribution factorizes into the $n$-fermion tree amplitude times a two-loop NLL factor. For instance, the factorizable diagrams associated with the fourth diagram in (2.1) are
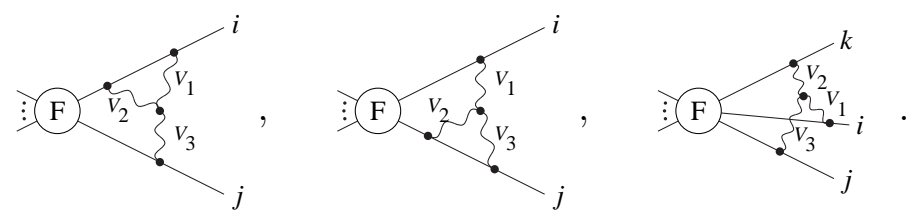

To extract the NLLs of soft/collinear origin, we exploit the fact that in the soft/collinear limit the coupling of a gauge boson $V$ to the $i$-th external fermion yields a factor $-2 e I_{i}^{V}\left(p_{i}+q\right)^{\mu}$, where $q$ and $p_{i}$ are the gauge-boson and fermion momenta, while $I_{i}^{V}$ is the gauge-group generator. For instance, for the last diagram in (2.2) we obtain

$$
\mu_{\mathrm{D}}^{4 \varepsilon} \int \frac{\mathrm{d}^{D} q_{1} \mathrm{~d}^{D} q_{2}}{(2 \pi)^{2 D}} \frac{-8 \mathrm{i} e^{3} g_{2} \varepsilon^{\bar{V}_{1} \bar{V}_{2} \bar{V}_{3}}\left[g_{\mu_{1} \mu_{2}}\left(q_{1}-q_{2}\right)_{\mu_{3}}+\text { perm. }\right] l_{1}^{\mu_{1}} l_{2}^{\mu_{2}} l_{3}^{\mu_{3}}}{\left(q_{1}^{2}-M_{V_{1}}^{2}\right)\left(q_{2}^{2}-M_{V_{2}}^{2}\right)\left(q_{3}^{2}-M_{V_{3}}^{2}\right)\left(l_{1}^{2}-m_{1}^{2}\right)\left(l_{2}^{2}-m_{2}^{2}\right)\left(l_{3}^{2}-m_{3}^{2}\right)} \times I_{i}^{V_{1}} I_{k}^{V_{2}} I_{j}^{V_{3}} \mathscr{M}_{0}
$$

where $q_{3}=-q_{1}-q_{2}, l_{1}=p_{i}-q_{1}, l_{2}=p_{k}-q_{2}$ and $l_{3}=p_{j}-q_{3}$. The $n$-fermion tree amplitude $\mathscr{M}_{0}$ must be regarded as a vector carrying SU(2) indices associated with the isospin of the scattering fermions, and the generators $I_{i}^{V}$ act as $\mathrm{SU}(2)$ matrices on the $i$-th fermion.

In addition to soft/collinear contributions, there are also two-loop NLL terms that arise from UV divergences in the one-loop sub-diagrams. In this case, the UV singularities are removed by means of a minimal subtraction at the scale $\mu^{2}=Q^{2}$. As a consequence, only those UV-divergent sub-diagrams characterized by scales of order $M^{2} \ll Q^{2}$ produce NLL terms. In particular, nonfactorizable diagrams do not produce NLL terms of UV origin. The explicit factorization of the NLL contributions of UV origin is obtained by means of projector techniques $[15,16]$ since the

\footnotetext{
${ }^{2}$ In these tree sub-diagrams, which are denoted with an 'F' in 2.2, the loop momenta must be set to zero. This follows from the collinear Ward identities.
} 
soft-collinear approximation is not applicable in this case. After the abovementioned minimal UV subtraction, we perform a finite renormalization that restores the on-shell normalization of the wave functions and translates the couplings to the minimal-subtraction scheme at the scale $\mu_{\mathrm{R}}$.

For processes involving heavy quarks, in addition to the contributions listed above also diagrams with Yukawa interactions contribute. Many of them are suppressed due to the behaviour of the interactions of scalar fields in the soft/collinear and $M \rightarrow 0$ limits. Only three types of diagrams are not suppressed,
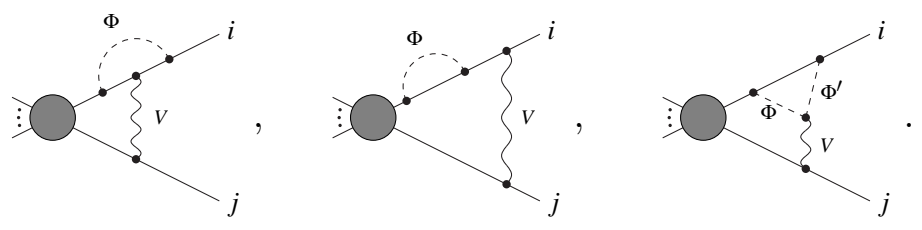

Moreover it turns out that the sum of the NLL terms resulting from these diagrams cancels owing to global gauge invariance of the Yukawa sector [16]. As a consequence, Yukawa interactions contribute only through fermionic self-energy diagrams that appear in the renormalization of the heavy-quark wave functions.

\section{High-energy expansion of the loop integrals}

The loop integrals have been calculated in the $Q^{2} \gg M^{2}$ limit to NLL accuracy using the sectordecomposition technique $[17,18]$ and, alternatively, the method of expansion by regions [19-22].

\subsection{Sector decomposition}

Sector decomposition is based on the Feynman-parameter representation. Exploiting general properties of soft, collinear and UV singularities, one can factorize these singularities in Feynmanparameter space in such a way that the divergent integrations assume a standard form. If all particles are massless these singular integrals are trivial and produce simple $\varepsilon$-poles [17]. In presence of massive particles, the general solution is available to NLL accuracy [18]. This permits to construct, in a completely automatized way, finite integral representations for the coefficients of all NLL terms of type $\varepsilon^{-k} \ln ^{j-k}\left(Q^{2} / M^{2}\right)$. The integrands are smooth and can easily be integrated numerically. ${ }^{3}$ Moreover, although these integrals do not have a standard form, it turns out that they are relatively simple. In practice they can be solved in analytic form by means of standard tricks (partial fractioning, integration by parts, etc.) combined with a table of elementary integrals. Also this part of the calculation is highly automatized, however, the set of integration rules might need to be extended when new diagrams are computed.

\subsection{Expansion by regions}

Within the expansion-by-regions method [19-22], the integration domain of the loop momenta is divided into regions corresponding to the asymptotic limit considered. The integrand is appropriately expanded in every relevant region, and each of the expanded terms is integrated over the whole integration domain. Each expanded term has a unique order in powers of $Q$ and $M$, but the

\footnotetext{
${ }^{3}$ This holds only in the unphysical region where all invariants $r_{i j}$ are negative, but allows for very useful checks.
} 
on-shell momenta $p_{i}$ of massive fermions involve two scales, $p_{i}^{2} \sim M^{2}$ and $2 p_{i} p_{j} \sim Q^{2}$. In order to separate these scales, the massive momenta are reparametrized in terms of light-like momenta $\tilde{p}_{i, j}$ as $p_{i}=\tilde{p}_{i}+p_{i}^{2} /\left(2 \tilde{p}_{i} \tilde{p}_{j}\right) \tilde{p}_{j}$. For the loop integrals needed in this calculation, the following regions for each loop momentum $k$ are relevant: hard, soft and ultrasoft regions, where $k^{2}$ is of the order $Q^{2}$, $M^{2}$ and $M^{4} / Q^{2}$, respectively; collinear, softcollinear and ultracollinear regions, where $k$ is collinear to one of the external momenta and $k^{2}$ is of the order $M^{2}, M^{4} / Q^{2}$ and $M^{6} / Q^{4}$, respectively. The latter two regions contribute only if the external fermions are massive. The expanded loop integrals have been evaluated using Mellin-Barnes representations, from which the extraction of NLLs has been automatized. In addition, Mellin-Barnes representations of the unexpanded integrals have been used to check the completeness of the set of regions used for the expansion.

\section{Results and conclusions}

The renormalized 2-loop NLL amplitude is expressed in terms of combinations of SU(2) matrices acting on the tree-level amplitude. This expression is simplified by means of $\mathrm{SU}(2)$ algebraic identities and additional relations resulting from global gauge invariance. The final result is consistent with the double-exponentiation formula ${ }^{4}$

$$
\mathscr{M}=\exp \left(\frac{\alpha}{8 \pi} \sum_{\substack{i, j=1 \\ j \neq i}}^{n} \delta_{i j}^{\mathrm{em}}\right) \exp \left(\frac{\alpha}{8 \pi} \sum_{\substack{i, j=1 \\ j \neq i}}^{n} \delta_{i j}^{\mathrm{sew}}\right)\left(1+\frac{\alpha}{8 \pi} \sum_{\substack{i, j=1 \\ j \neq i}}^{n} \delta_{i j}^{Z}\right) \mathscr{M}_{0}
$$

The term $\delta_{i j}^{\text {sew }}$ behaves as if all electroweak gauge bosons would have the same mass $M$ and is symmetric with respect to $\mathrm{SU}(2) \times \mathrm{U}(1)$ transformations,

$$
\delta_{i j}^{\mathrm{sew}}=-\sum_{V=B, W^{a}} I_{i}^{\bar{V}} I_{j}^{V} I\left(\varepsilon, M,-r_{i j}\right)-\frac{1}{(4 \pi)^{2}}\left(g_{1}^{2} \frac{Y_{i} Y_{j}}{4} b_{1}^{(1)}+g_{2}^{2} T_{i}^{a} T_{j}^{a} b_{2}^{(1)}\right) J\left(\varepsilon, M, \mu_{\mathrm{R}}^{2}\right) .
$$

Here $T_{i}^{a}$ and $Y_{i}$ are the generators of the $\mathrm{SU}(2) \times \mathrm{U}(1)$ group, $g_{1,2}$ are the corresponding coupling constants, and $b_{1,2}^{(1)}$ the one-loop $\beta$-function coefficients [15]. The NLL functions read

$$
I(\varepsilon, M, r)=-L^{2}-\frac{2}{3} L^{3} \varepsilon-\frac{1}{4} L^{4} \varepsilon^{2}+\left[\frac{3}{2}-\ln \left(\frac{r}{Q^{2}}\right)-\frac{y_{i}^{\kappa_{i}}}{C_{i}^{\mathrm{ew}}} \frac{g_{2}^{2} m_{\mathrm{t}}^{2}}{8 e^{2} M_{\mathrm{W}}^{2}}\right]\left(2 L+L^{2} \varepsilon+\frac{1}{3} L^{3} \varepsilon^{2}\right)
$$

and $J\left(\varepsilon, M, \mu_{\mathrm{R}}^{2}\right)=\left[I\left(2 \varepsilon, M, Q^{2}\right)-\left(Q^{2} / \mu_{\mathrm{R}}^{2}\right)^{\varepsilon} I\left(\varepsilon, M, Q^{2}\right)\right] / \varepsilon$ with $L=\ln \left(Q^{2} / M^{2}\right) . \operatorname{In}(4.3) C_{i}^{\mathrm{ew}}=$ $\sum_{V} I_{i}^{\bar{V}} I_{i}^{V}$, and the Yukawa terms proportional to $m_{\mathrm{t}}^{2}$ contribute with factors $y_{\mathrm{t}, \mathrm{b}}^{\mathrm{L}}=1, y_{\mathrm{t}}^{\mathrm{R}}=2, y_{\mathrm{b}}^{\mathrm{R}}=0$, for heavy quarks $i=\mathrm{b}, \mathrm{t}$ with chirality $\kappa_{i}=\mathrm{R}, \mathrm{L}$, and $y_{i}^{K}=0$ otherwise. The term $\delta_{i j}^{\mathrm{em}}$ in (4.1) depends on the $\gamma-\mathrm{W}$ mass gap and contains all soft/collinear singularities due to massless photons. These singularities involve single and double $\varepsilon$-poles. Thus the terms $\delta_{i j}^{\text {sew }}$ and $\delta_{i j}^{Z}$ in (4.1) need to be expanded up to $\mathscr{O}\left(\varepsilon^{2}\right)$. We find that $\delta_{i j}^{\text {em }}$ behaves as in QED [16]. Finally we find that all contributions depending on the $\mathrm{Z}-\mathrm{W}$ mass difference can be factorized into the one-loop term $\delta_{i j}^{Z}=-I_{i}^{Z} I_{j}^{Z} \ln \left(M_{\mathrm{Z}}^{2} / M_{\mathrm{W}}^{2}\right)\left[2 L+2 L^{2} \varepsilon+L^{3} \varepsilon^{2}\right]$. Apart from these latter $\ln \left(M_{\mathrm{Z}}^{2} / M_{\mathrm{W}}^{2}\right)$ terms, all effects of symmetry breaking (terms proportional to $M_{\mathrm{W}} / M_{\mathrm{Z}}$, mixing parameters and vacuum expectation

\footnotetext{
${ }^{4}$ In the following we set $\mu_{\mathrm{D}}^{2}=Q^{2} e^{\gamma_{\mathrm{E}}} /(4 \pi)$.
} 
value) disappear due to non-trivial cancellations between different Feynman diagrams. These cancellations originate from relations between the gauge-boson masses, the mixing parameters, and the vacuum expectation value. The result is in agreement with the resummation prescriptions proposed for massless $[6,8]$ and massive fermions [7] and exhibits strict analogies with the general form of two-loop singularities in massless QCD [23].

\section{References}

[1] J. H. Kühn, A. Kulesza, S. Pozzorini and M. Schulze, Nucl. Phys. B 727 (2005) 368 [hep-ph/0507178].

[2] J. H. Kühn, A. Kulesza, S. Pozzorini and M. Schulze, arXiv:0708.0476 [hep-ph].

[3] A. Denner and S. Pozzorini, Eur. Phys. J. C 18 (2001) 461 [hep-ph/0010201].

[4] A. Denner and S. Pozzorini, Eur. Phys. J. C 21 (2001) 63 [hep-ph/0104127].

[5] V. S. Fadin, L. N. Lipatov, A. D. Martin and M. Melles, Phys. Rev. D 61 (2000) 094002 [hep-ph/9910338].

[6] J. H. Kühn, A. A. Penin and V. A. Smirnov, Eur. Phys. J. C 17 (2000) 97 [hep-ph/9912503].

[7] M. Melles, Phys. Rept. 375 (2003) 219 [hep-ph/0104232].

[8] J. H. Kühn, S. Moch, A. A. Penin and V. A. Smirnov, Nucl. Phys. B 616 (2001) 286 [Erratum-ibid. B 648 (2003) 455] [hep-ph/0106298].

[9] J. H. Kühn, F. Metzler and A. A. Penin, arXiv:0709.4055 [hep-ph].

[10] J. y. Chiu, F. Golf, R. Kelley and A. V. Manohar, arXiv:0712.0396 [hep-ph].

[11] W. Beenakker and A. Werthenbach, Nucl. Phys. B 630 (2002) 3 [hep-ph/0112030].

[12] A. Denner, M. Melles and S. Pozzorini, Nucl. Phys. B 662 (2003) 299 [hep-ph/0301241].

[13] S. Pozzorini, Nucl. Phys. B 692 (2004) 135 [hep-ph/0401087].

[14] B. Jantzen, J. H. Kühn, A. A. Penin and V. A. Smirnov, Nucl. Phys. B 731 (2005) 188 [Erratum-ibid. B 752 (2006) 327] [hep-ph/0509157].

[15] A. Denner, B. Jantzen and S. Pozzorini, Nucl. Phys. B 761 (2007) 1 [hep-ph/0608326].

[16] A. Denner, B. Jantzen and S. Pozzorini, in preparation.

[17] T. Binoth and G. Heinrich, Nucl. Phys. B 680 (2004) 375 [hep-ph/0305234].

[18] A. Denner and S. Pozzorini, Nucl. Phys. B 717 (2005) 48 [hep-ph/0408068].

[19] M. Beneke and V. A. Smirnov, Nucl. Phys. B 522 (1998) 321 [hep-ph/9711391].

[20] V. A. Smirnov and E. R. Rakhmetov, Theor. Math. Phys. 120 (1999) 870 [hep-ph/9812529].

[21] V. A. Smirnov, Phys. Lett. B 465 (1999) 226 [hep-ph/9907471].

[22] B. Jantzen and V. A. Smirnov, Eur. Phys. J. C 47 (2006) 671 [hep-ph/0603133].

[23] S. Catani, Phys. Lett. B 427 (1998) 161 [hep-ph/9802439]. 